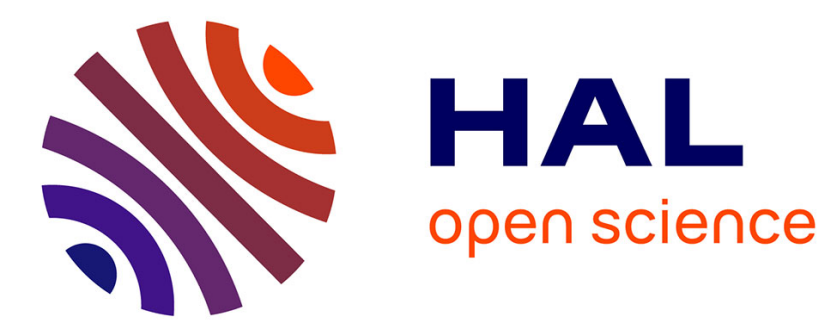

\title{
Best-effort Highway Traffic Congestion Control via Variable Speed Limits
}

Carlos Canudas de Wit

\section{To cite this version:}

Carlos Canudas de Wit. Best-effort Highway Traffic Congestion Control via Variable Speed Limits. CDC 2011 - ECC 2011 - 50th IEEE Conference on Decision and Control and European Control Conference, Dec 2011, Orlando, Floride, United States. pp.s/n. hal-00642038

\section{HAL Id: hal-00642038 \\ https://hal.science/hal-00642038}

Submitted on 17 Nov 2011

HAL is a multi-disciplinary open access archive for the deposit and dissemination of scientific research documents, whether they are published or not. The documents may come from teaching and research institutions in France or abroad, or from public or private research centers.
L'archive ouverte pluridisciplinaire HAL, est destinée au dépôt et à la diffusion de documents scientifiques de niveau recherche, publiés ou non, émanant des établissements d'enseignement et de recherche français ou étrangers, des laboratoires publics ou privés. 


\title{
Best-effort Highway Traffic Congestion Control via Variable Speed Limits
}

\author{
Carlos Canudas de Wit*
}

\begin{abstract}
The problem of controlling the congestion front in a single link road section is considered in this paper. For this purpose, we introduce a new variable-length two-cell lumped model composed of; one congested cell, and another in free flow. This model has the advantage of having few states while preserving the vehicle conservation property. This model is used as a basis to design a simple "best-effort" controller that regulates (at its best) the congestion front to some prespecified value. The control law can be implemented using only information about the congestion front position.
\end{abstract}

\section{INTRODUCTION}

The front congestion control problem consists at regulating the front congestion to some pre-specified value in order to avoid that the congestion overspread upstream blocking other exit ramps (producing even largest congestion conditions), or/and reaching critical safety sections (i.e. tunnels, intersections, etc.).

In the traffic control literature, the control can be formulated either by ramp metering regulation [4], [5], [7], [8], [9], or by variable speed limit control [6], [13]. The first method aims at regulating the inflow on some (or several) input ramps of the road. This control setup is highly effective in regulating flows and densities on the main lane, at the price of reallocate the vehicles distribution into other parts of the network (usually at upstream location of the considered network where demands are lowers). A complete overview on ramp metering strategies can be found in [15]. The second method consists in regulating the speed limits. By this mean, the maximum capacity of the regulated section can be modified. Lowering the speed limits will results in a reduction of the road maximum capacity. This will reduce the grown rate of the congestion front spreading, but it will increase the traveling time along the free section. It is also possible to envision the combination of both control strategies, as reported in recent results [1], [14], [16].

In this paper we consider the problem of controlling the congestion front in a single link highway section using variable speed control. To this aim, we propose a new variable-length two-cell lumped model, composed of one congested cell and another in free flow. Compared to existing multi-cell models with constant dimension, this model has the advantage of having only few states ( 3 in total including: 2 density states for the congested and the free cells, and one more for the evolution of the congestion front) The model is build such that the cells are variable in length while preserving the vehicle conservation property.

\footnotetext{
*Director of reearch at the CNRS, GIPSA-Lab. NeCS team, Grenoble, France. carlos.canudas-de-witegipsa-lab.inpg.fr
}

The variable-length two-cell lumped model model is used as a basis to design a simple "best-effort" controller that regulates (at its best) the congestion front to some pre-specified value. The control is designed under constraints concerning magnitude step changes, and dwell-time. The control law discrete-time implementation only needs information on the congestion front position. This information can be estimated directly from camera sensors networks, or indirectly by building a density observed based on the proposed variablelength model. This issues will not be treated in this paper, and are under current investigation.

The paper first recall the derivation grounds of the LWR model with constant and multiple cells, then we introduce our new two-cell variable-length model. The subsequent sections presents the best-effort control design, and report some simulations.

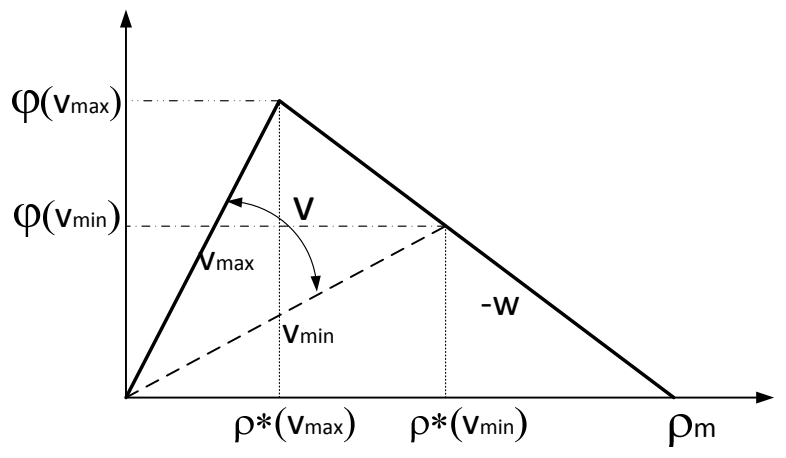

Fig. 1. Variation of the fundamental diagram as a function of the speed limit $v$; the maximum capacity $\varphi_{m}=\varphi\left(v_{\max }\right)$ is reach for the maximum available velocity $v_{\max }$, at the critical density $\rho^{*}\left(v_{\max }\right)$. The capacity of the section will decrease to $\varphi_{m}=\varphi\left(v_{\min }\right)$ when the speed limit is set to its minimum $v_{\min }$. Nevertheless the critical density is increased substantially $\rho^{*}\left(v_{\min }\right)$

\section{Multiple-Cells, CONSTANT-Length LWR MOdeL}

The traffic dynamics models are based on the vehicle conservation principle. The simplest continuous macroscopic traffic model, involving only the density $\rho$, is the LWR cell transmission model introduced in [11], [17]. It is also known under the name of cell transmission model, and it has been shown to be consistent with hydrodynamic theory [2]. Validation tests with real data have been reported in [12]. The constitutive assumption of this model, motivated by experimental data, is that the vehicles tend to travel at an equilibrium speed $v=v(\rho)$, where $\rho$ represents the density of a specific section at a specific time. 
In variable speed limit control the velocity, $v$, in the decongested cell, becomes the main control input that can be actuated (under the assumption that drivers will respectin average-the suggested speed limits) using variable sign panels located at the road side.

The equilibrium speed depends implicitly on the location and on the time. Since the flow is defined as $\varphi(\rho)=\rho v(\rho)$, one can depict an equilibrium flow function $\varphi=\varphi(\rho)$ called the fundamental diagram. As shown in Fig. 1, the fundamental diagram can be defined, in its simplest form, as a triangle with its maximum at $\varphi_{m}=\varphi\left(\rho^{*}\right)$ describing the maximum capacity of the road. The critical density $\rho^{*}$ defines the boundary between the decongested and the congested zones. $\rho_{m}$ is the maximum density that the road can support. The slope $-w$ defines the speed at which congestion will travel upstream.

If the speed limits are changed during operation, the fundamental diagram will be affected as show Fig. 1: a decrease in $v$ will reduce the maximum road capacity $\varphi_{m}$ but will increase the critical density $\rho^{*}$. The net effect of this action will be that congestions grown rate will be reduced. If a great portion of the road is congested then the total traveling time may be also improved. Inversely, if the majority of the road is decongested, then the traveling time may be increased. In cases (not studied here) when an exit ramp is block due to the congestion arrival, further potential improvements may be expected.

The evolution of the number of vehicles within any spatial section $(0, L)$, is given by the following car conservation law in term of the number of vehicles, $N$, in the cell ${ }^{1}$ :

$$
\frac{\mathrm{d}}{\mathrm{d} t} N=\varphi_{\text {in }}-\varphi_{\text {out }}, \quad N=\int_{0}^{L} \rho(x, t) \mathrm{d} x
$$

where $\varphi_{\text {in }}$ and $\varphi_{\text {out }}$ are the input (at $x=L$ ) and output (at $x=0$ ) flows at the boundaries of the road section.

Consider now that the road section is divided in $n$-cells of constant length $l_{i}$. Let us denote by $\rho_{i}$ density of the $i^{\text {th }}$ cell of the section. Then, the number of vehicles per cell is noted as $N_{i}=\rho_{i} l_{i}$.

As conservation laws generate irregular flows, they cannot be integrated numerically using standard methods (see [10], [2]). An efficient first-order numerical method to treat such conservation laws is the Godunov scheme [3] which is a first order scheme that reproduce correctly the propagation of the shock waves avoiding oscillating behavior and having a physical interpretation. Using the Godunov mathematical formalism, the conservation law (2) takes the following discrete representation

\footnotetext{
${ }^{1}$ Equation (1) can be rewritten (see [11]) as a hyperbolic equation involving only the density:

$$
\partial_{t} \rho+\partial_{x} \varphi(\rho)=0
$$

The macroscopic continuous density dynamics is then given by the LWR Cauchy problem described by (1) with the initial condition $\rho(x, 0)=\rho^{0}(x)$.
}

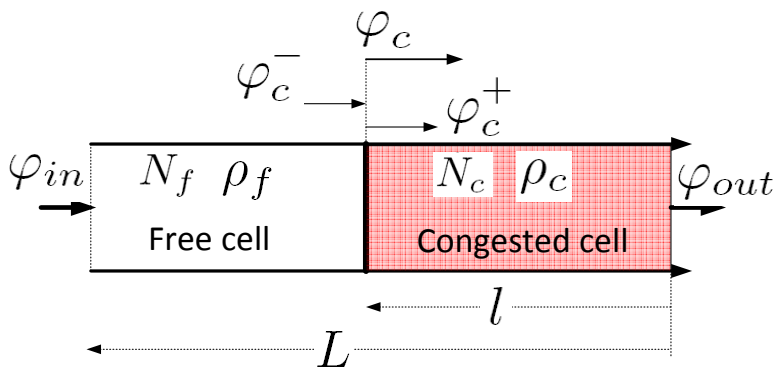

Fig. 2. Schematic diagram of the two-cell variable-length model.

$$
\rho_{i}(k+1)=\rho_{i}(k)+\frac{T}{l_{i}}\left(\varphi_{i}(k)-\varphi_{i+1}(k)\right)
$$

where $k$ is the time index, $T$ is the discrete time interval ${ }^{2}$, and $\varphi_{i}$ is the interface flow between the cells $i-1$ and $i$ given as:

$$
\varphi_{i}=\min \left\{D_{i-1}, S_{i}\right\}
$$

with

$$
\begin{aligned}
D_{i-1} & =\min \left\{v_{i-1} \rho_{i-1}, \varphi_{m, i-1}\right\}, \\
S_{i} & =\min \left\{\varphi_{m, i}, w_{i}\left(\rho_{m, i}-\rho_{i}\right)\right\}
\end{aligned}
$$

where the demand $D_{i-1}$ is the flow that can be delivered by the cell $i-1$ to the cell $i$ while the supply $S_{i}$ is the flow that can be received by the cell $i$ from the cell $i-1$. $\varphi_{m, i}$ is the maximum flow allowed by the capacity of cell $i$, $\rho_{m, i}$ is the jam density (i.e. the maximum density that can be reached), $v_{i}$ corresponds to the free flow speed and $w_{i}$ is the congestion wave speed in cell $i$. All these parameters can be the same for all the cells or allowed to vary for each cell. In variable speed control, the value of $v_{i}$ may be modified for each cell, or for a group of them.

\section{A NEW TWO-CELL VARIABLE-LENGTH MODEL}

For purposes of controlling the congestion front of a road section, we consider a section split into two cells of variable length; a congested downstream cell of length $l=l(t)$, and an decongested (free flow) one of length $L-l$, where $L$ is the total length of the considered section, see Fig. 1.

\section{A. Vehicle conservation law}

Let introduce the number of vehicles in the free $\left(N_{f}\right)$ and in the congested $\left(N_{c}\right)$ cells as lumped quantities

$$
\begin{aligned}
N_{f} & =(L-l) \rho_{f} \\
N_{c} & =l \rho_{c}
\end{aligned}
$$

being $\rho_{f}$, and $\rho_{c}$ the associated (lumped) densities. Following the conservation law (2), and using the flows boundaries as

\footnotetext{
${ }^{2}$ The condition $v T<l$, with $v$ the free-flow speed, is a sufficient condition for (3) to converge.
} 
indicated by the Godunov formalism, we get the following model

$$
\begin{aligned}
\dot{N}_{f} & =\min \left\{\varphi_{\text {in }}, S_{f}\right\}-\min \left\{D_{f}, S_{c}\right\} \\
\dot{N}_{c} & =\min \left\{D_{f}, S_{c}\right\}-\min \left\{D_{c}, \varphi_{\text {out }}\right\}
\end{aligned}
$$

where the demand and supply functions for the free flow cell is given as

$$
\begin{aligned}
D_{f} & =\min \left\{v_{f} \rho_{f}, \varphi_{m}\left(v_{f}\right)\right\} \\
S_{f} & =\min \left\{\varphi_{m}\left(v_{f}\right), w_{f}\left(\rho_{m}-\rho_{f}\right)\right\}
\end{aligned}
$$

while the demand and supply function for the congested cell are give by

$$
\begin{aligned}
D_{c} & =\min \left\{v_{c} \rho_{c}, \varphi_{m}\left(v_{c}\right)\right\} \\
S_{c} & =\min \left\{\varphi_{m}\left(v_{c}\right), w_{c}\left(\rho_{m}-\rho_{c}\right)\right\}
\end{aligned}
$$

$\varphi_{\text {in }}$, and $\varphi_{\text {out }}$ are the input and output flows respectively. Note that in this formulation, the maximal capacity $\varphi_{m}(\cdot)$, is a function of the free flow velocity $v$ that will play the role of the control input in the subsequent formulation.

As mentioned before, it is assumed that the downstream cell is congested while the upstream cell is free. This implies that:

$$
\begin{aligned}
0 \leq \rho_{f} & \leq \rho^{*} \\
\rho^{*}<\rho_{c} & \leq \rho_{m}
\end{aligned}
$$

From the these equations, and assuming that the input and output flows are below the maximum admissible ones, i.e. $\varphi_{\text {in }} \leq \varphi_{m}\left(v_{f}\right), \varphi_{\text {out }} \leq \varphi_{m}\left(v_{c}\right)$, then we have:

$$
\begin{aligned}
\min \left\{\varphi_{\text {in }}, S_{f}\right\} & =\varphi_{\text {in }} \\
\min \left\{D_{c}, \varphi_{\text {out }}\right\} & =\varphi_{\text {out }}
\end{aligned}
$$

Therefore model (5)-(6) simplifies to:

$$
\begin{aligned}
\dot{N}_{f} & =\varphi_{\text {in }}-\varphi_{n}(v) \\
\dot{N}_{c} & =\varphi_{n}(v)-\varphi_{\text {out }}
\end{aligned}
$$

where

$$
\varphi_{n}(v)=\varphi_{n}\left(v_{f}, v_{c}\right)=\min \left\{D_{f}, S_{c}\right\}
$$

with $n=1,2$, i.e.

$$
\begin{array}{ll}
\varphi_{1}(v) \\
\varphi_{2}(v)
\end{array}= \begin{cases}\min \left\{v_{f} \rho_{f}, \varphi_{m}\left(v_{f}\right)\right\} & \text { if, } D_{f}<S_{c} \\
\min \left\{\varphi_{m}\left(v_{c}\right), w_{c}\left(\rho_{m}-\rho_{c}\right)\right\} & \text { else }\end{cases}
$$

The model has then operational modes. When $n=1$ the model is say to be in the "absorption" phase as in this condition, $D_{f}<S_{c}$, the congested cell is able to absorb the arriving flow making the congestion to decrease. Inversely, the when $n=2$, there is an surplus of arriving flow at the congestion front, $D_{f} \geq S_{c}$. The model is then say to be at the "expansion" phase, as the congestion will tends to expand upstream. The difference between $D_{f}$, and $S_{c}$ will be used as a basis to build a dynamics of the congestion front.

\section{B. Dynamics of the congestion front}

Note that at this point, the control variable $v$ does not explicitly appear in the vehicle conservation model above. This will happen when the variable $N$ will be replaced by its relation with the density and associated length. To this aim, the conservation model above needs to be completed with another equation describing the evolution of the congestion position $l$.

The proposed variation law for $l$ is

$$
\begin{aligned}
i= & c\left(\varphi_{c}^{-}-\varphi_{c}^{+}\right)=c\left(D_{f}-S_{c}\right) \\
= & c\left(\min \left\{v_{f} \rho_{f}, \varphi_{m}\left(v_{f}\right)\right\}-\right. \\
& \left.-\min \left\{\varphi_{m}\left(v_{c}\right), w_{c}\left(\rho_{m}-\rho_{c}\right)\right\}\right)
\end{aligned}
$$

where $c[\mathrm{~m} /$ Vehicle $]$ is a constant describing the mean spatial occupance per vehicle on the section. It can be approximated from the maximum density as $c \approx 1 / \rho_{m}$. The equation describe the growing rate of the congestion as the product between the difference between left $\left(\varphi_{c}^{-}=\varphi_{f}\right)$ and right $\left(\varphi_{c}^{+}=\varphi_{c}\right)$ flows at the congestion line, and the constant $c$.

Congestion will increase when the flow at the free cell is greater than the flow at the congested one, i.e. $\varphi_{c}^{-}>\varphi_{c}^{+}$, and it will decrease otherwise.

Remark 1 A parallel with physical law can be made by understanding equation (9) as a force law produced as a consequence of the (integral of) the differential pressure (stress) between neighborhood cells. The constant c can be seen as the stiffness of the diaphragm supporting this stress.

Remark 2 In the context of traffic engineering, the equation (9) can be seen as a simplification of the Rankine-Hugoniot condition, that specify that the shock speed can be written as

$$
\dot{s}=\frac{\varphi\left(\rho^{+}\right)-\varphi\left(\rho^{-}\right)}{\rho^{+}-\rho^{-}}=\frac{1}{\rho^{+}-\rho^{-}} \int_{\rho^{-}}^{\rho^{+}} \varphi^{\prime}(\psi) d \psi
$$

in the context of distributed PDEs models, where $\rho^{+}, \rho^{-}$are the right/left densities at the from congestion line, respectively. Equation (9) is intended for a lumped model that will be used for control synthesis, as show latter in the paper.

\section{Implicit and explicit full model forms}

The following hypothesis are adopted:

$H 1)$ The whole section has the same fundamental diagram. That is; $v=v_{c}=v_{f}$, and $w=w_{c}=w_{f}$, where $v=$ $v(t)$ is time-varying but $w$ is assumed to be constant.

H2) The critical density $\rho^{*}(v)$, and its associated road maximum capacity $\varphi_{m}(v)$, are both functions of $v$,

$$
\rho^{*}=\rho^{*}(v)=\frac{w \rho_{m}}{v+w}, \quad \varphi_{m}(v)=v \rho^{*}=v \frac{w \rho_{m}}{v+w}
$$

but the maximum density $\rho_{m}$ is independent of $v$, see Figure 1.

Noticing that $N=\rho \cdot l$, we have that

$$
\dot{N}_{c}=l \dot{\rho}_{c}+i \rho_{c}, \quad \dot{N}_{f}=(L-l) \dot{\rho}_{f}-i \rho_{f}
$$


the implicit form of model (2)-(9) writes as:

$$
\begin{aligned}
\dot{\rho}_{f}= & \frac{1}{L-l}\left(\varphi_{\text {in }}-\varphi_{n}(v)+i_{\rho_{f}}\right) \\
\dot{\rho}_{c}= & \frac{1}{l}\left(\varphi_{n}(v)-\varphi_{\text {out }}-i \rho_{c}\right) \\
i= & c\left(\min \left\{v \rho_{f}, \varphi_{m}(v)\right\}-\right. \\
& \left.-\min \left\{\varphi_{m}(v), w_{c}\left(\rho_{m}-\rho_{c}\right)\right\}\right)
\end{aligned}
$$

where $\varphi_{\text {in }}, \varphi_{\text {out }}$ are exogenous inputs, $\left(\rho_{f}, \rho_{c}, l\right)$ are the state variables, and $v$ is the control input. It should be noted that this is a lumped and highly nonlinear model. The model implicitly assume a separation between the free and congested cell, i.e. $\rho_{f} \in\left[0, \rho^{*}(v)\right], \rho_{c} \in\left[\rho^{*}(v), \rho_{m}\right]$.

The explicit version of this model including explicit saturation functions can be introducing in the model by using the relation $\operatorname{Sat}_{0}^{a}(x)=\min \{a, x\}, \forall, a>0, x \geq 0$,

$$
\begin{aligned}
\dot{\rho}_{f} & =\frac{1}{L-l}\left(\varphi_{\text {in }}-\bar{\varphi}_{n}(v)+i \rho_{f}\right) \\
\dot{\rho}_{c} & =\frac{1}{l}\left(\bar{\varphi}_{n}(v)-\varphi_{\text {out }}-i \rho_{c}\right) \\
i & =c\left(\operatorname{Sat}_{0}^{\varphi_{m}(v)}\left\{v \rho_{f}\right\},-\operatorname{Sat}_{0}^{\varphi_{m}(v)}\left\{w\left(\rho_{m}-\rho_{c}\right)\right\}\right)
\end{aligned}
$$

with

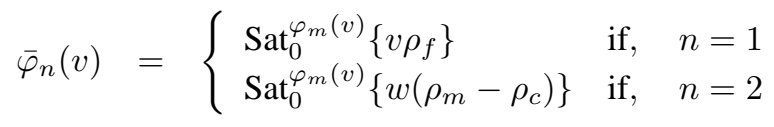

this version of the model makes explicitly the role of the control law $v$ in the model; its change road maximum capacity $\varphi_{m}(v)$, and the slope of the demand function of the free cell.

\section{FRONT-CONGESTION REGULATION VIA VARIABLE SPEED-LIMIT CONTROL}

The problem of front-congestion regulation control via variable speed-limit consists in finding a control law for $v$, function of the model states, such that the front congestion $l$ can be regulated around the reference value $l_{r}$.

In this section we present several options for this design.

\section{A. Best effort control}

Let $\tilde{l}=l-l_{r}$, then using $V=\tilde{l}^{2} / 2$, a Lyapunov-like control law for $v$ can be derived as the one that set

$$
D_{f}(v)-S_{c}+\frac{k}{c} \tilde{l}=0
$$

leading to $\dot{V}=-k \tilde{l}^{2}$.

Nevertheless if the speed limits are constrained to live in the set $\mathbb{U}=\left\{v_{\max }, v_{\min }\right\}$, then the best-effort constrained control with respect to the metric $V$, will be

$$
v *=\min _{v \in \mathbb{U}}\left|D_{f}(v)-S_{c}+\frac{k}{c} \tilde{l}\right|
$$

This problem can be solved graphically as shown in Fig. 3, and leads to the solution

$$
v *=\operatorname{Sat}_{v_{\min }}^{v_{\max }}\left(\frac{1}{\rho_{f}}\left[-w \rho_{c}+w \rho_{m}-\frac{k}{c}\right]\right)
$$

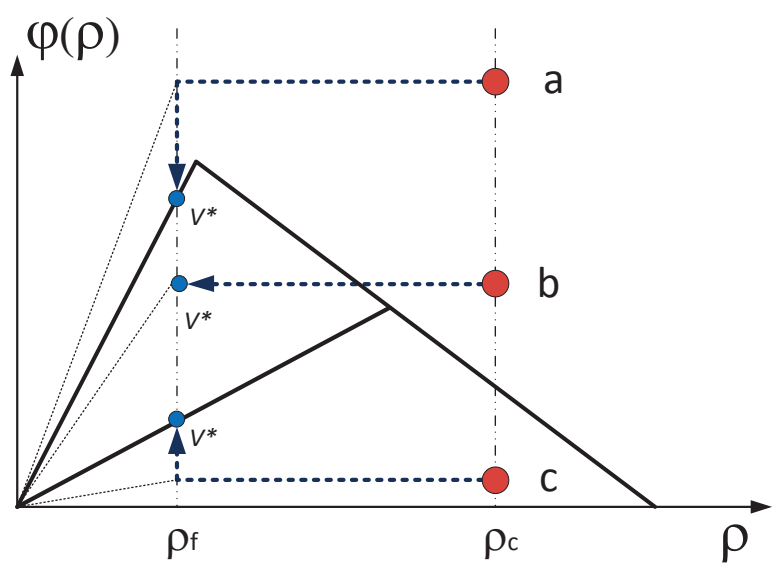

Fig. 3. Illustration of the optimal solution given by Equation (15). Three cases are considered for illustration: a) the point $a$ corresponds to the case where $S_{c}-\frac{k}{c} \tilde{l} \geq \sup _{v} \max _{\rho}\{\varphi(\rho)\}$, the optimal solution is projected on the upper boundaries of the admissible set of solutions $\mathbb{U}$, b) the point $b$ corresponds case where $\inf _{v} \min _{\rho}\{\varphi(\rho)\}<S_{c}-\frac{k}{c} \tilde{l}<$ $\sup _{v} \max _{\rho}\{\varphi(\rho)\}$, the optimal solution is $\frac{1}{\rho_{f}}\left[-w \rho_{c}+w \rho_{m}-\frac{k}{c} \tilde{l}\right]$, c) the point $c$ corresponds to the case where $S_{c}-\frac{k}{c} \tilde{l} \leq \inf _{v} \min _{\rho}\{\varphi(\rho)\}$, the optimal solution is projected on the lower boundaries of the admissible set of solutions $\mathbb{U}$.

\section{B. Best-effort control under dynamics and magnitude con- straints}

An alternative to the previous continuous-time state feedback law, is to derive a variation law for $v$ with a prescribed Dwell-time $T_{s}$, and a maximum steep variation $\Delta_{v}$. This requirement is motivated by constraints in the actuated variable speed signal (variable speed limit actuation is more likely to change by steps of several $K m / h$ ). This implies that the variation of the control $v$ should be constraint to change as,

$$
\nabla v(k)=v(k+1)-v(k) \in \mathbb{V}
$$

where $\mathbb{V}$ is the finite 3-valued set defined as

$$
\mathbb{V}=\left\{-\Delta_{v}, 0, \Delta_{v}\right\}
$$

Let now $V(k)=\tilde{l}^{2}(k)$, and $i$ be approximated as:

$$
l(k+1)=l(k)+c T_{s}\left(D_{f}(v(k))-S_{c}(k)\right)
$$

The constrained best-effort law can be derived by observing, with $\gamma(v(k), k)=c T_{s}\left(D_{f}(v(k))-S_{c}(k)\right)$, that

$$
\begin{aligned}
V(k+1)-V(k) & =\tilde{l}^{2}(k+1)-\tilde{l}^{2}(k) \\
& =2 \tilde{l} \gamma(v(k), k)+\gamma^{2}(v(k), k)
\end{aligned}
$$

As the rate of change of $v(k)$ is constrained to only 3-values in $\mathbb{V}$, the magnitude of the demand $D_{f}(v(k))$, and hence the magnitude of $\gamma(v(k), k)$, cannot be arbitrarily set to make $V(k)$ to decrease uniformly. In this context, the best effort control is

$$
v^{*}=\min _{v \in \mathbb{U}, \nabla v \in \mathbb{V}}\left\{2 \tilde{l} \gamma(v(k), k)+\gamma^{2}(v(k), k)\right\}
$$




\begin{tabular}{|l|c|c|c|}
\hline Cases & $\operatorname{sign}(\gamma(v(k), k))$ & $\operatorname{sign}(\tilde{l}(k))$ & $\nabla v(k)$ \\
\hline \hline$a)$ & 1 & -1 & 0 \\
\hline$b)$ & 1 & 1 & $-\Delta_{v}$ \\
\hline$c)$ & -1 & 1 & 0 \\
\hline$d)$ & -1 & -1 & $\Delta_{v}$ \\
\hline
\end{tabular}

TABLE I

SET OF BEST POSSIBLE SOLUTIONS OF THE CONTROL LAW (16)

which is indeed an optimization problem having constraints implying past values of the decision variable $v(k)$. Due above described limitations on the admissible values for $v(k)$, and hence for $\gamma$, a relaxation of this problem is possible by making the minimization problem $\gamma$-size insensitive, i.e. making the best possible choice for $v(k)$ so as sign of $\gamma$ be, when possible, opposed to the one of $\tilde{l}$,

$$
\min _{v \in \mathbb{U}, \nabla v \in \mathbb{V}}|\operatorname{sign}(\tilde{l})+\operatorname{sign}(\gamma(v(k), k))|
$$

an approximated solution of this problem results in

$$
v(k+1)=\operatorname{Sat}_{v_{\min }}^{v_{\max }}\left(v(k)-\frac{\Delta_{v}}{2}[\operatorname{sign}(\gamma(v(k), k))+\operatorname{sign}(\tilde{l})]\right)
$$

the rationality of this solution can be explained by looking the Table I, where four cases can be identified:

The first two cases $(a)-(b)$ correspond to situations where the demand in the free cell is greater than the supply at the congested cell, implying a grow in the congestion-front, i.e. $i>0$ as show Eq.-(12). In this case, the "best control action" is:

a) to keep the speed limits constant, $(\nabla v=0)$ when the front congestion is below the reference value $\left(l<l_{r}\right)$, and

b) to take a corrective action by reducing the speed limit velocity by an amount $\Delta_{v}$ during a time period $T_{s}$, when the front congestion is above the reference value $\left(l>l_{r}\right)$.

The last two cases $(c)-(d)$ concerns situations where the congestion front is likely to decrease i.e. $i<0$. In this case, the "best control action" is:

$c)$ to keep the speed limits constant $(\nabla v=0)$, if the congestion front is already larger than its reference $\left(l>l_{r}\right)$, and

d) to increase the speed limits $\left(\nabla v=\Delta_{v}\right)$ if the congestion front is below its reference value $\left(l<l_{r}\right)$.

\section{Implementation issues}

It is worth to note that the implementation of this controller requires the measure of the sign of the error distance $\tilde{l}$, and the sign of $\gamma$. From Eq.(12) we see that $\operatorname{sign}(\gamma)=\operatorname{sign}(i)$, therefore, the control law (16) has the following alternative representation, which simplify its implementation

$$
\begin{aligned}
v(k+1) & =\operatorname{Sat}_{v_{\min }}^{v_{\max }}(v(k)- \\
& \left.-\frac{\Delta_{v}}{2}[\operatorname{sign}(l(k+1)-l(k))+\operatorname{sign}(\tilde{l}(k))]\right)
\end{aligned}
$$

Then, by measuring only $l(k)$, and the above control can be implemented.
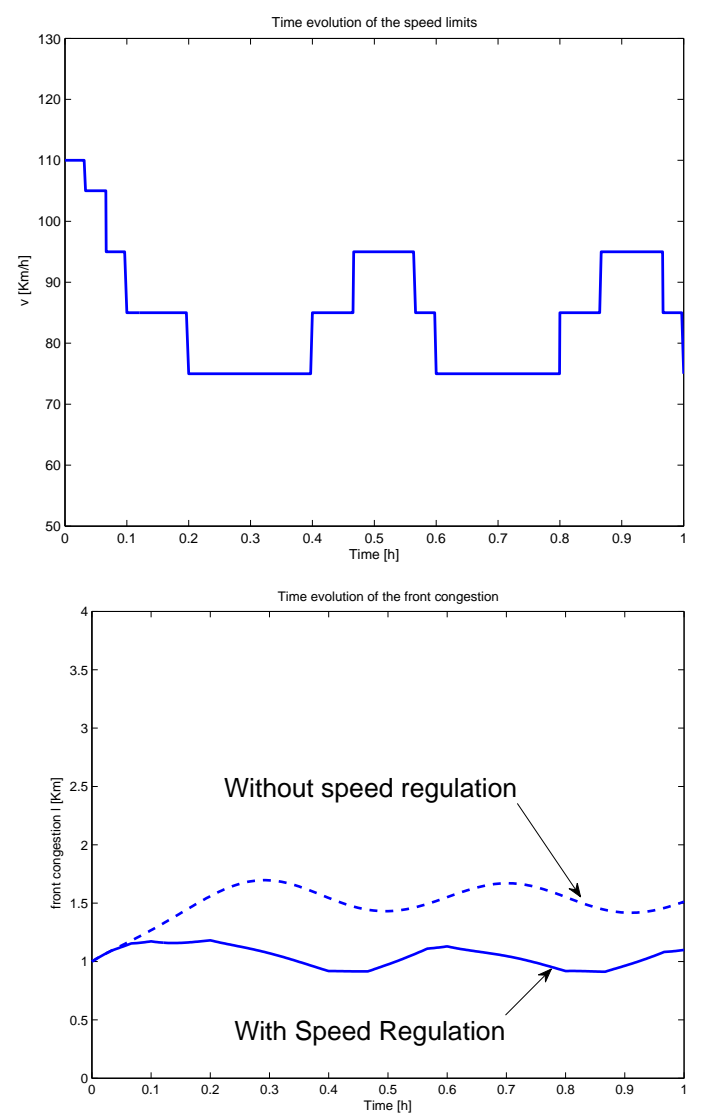

Fig. 4. Congestion front regulation via variable speed limit. The upper figure shows the time-evolution of the control $v$. The lower figures compares the line front $l$ obtained using variable-speed limit control (continuous line), to the one obtained without regulation (dashed lines).

\section{Simulation Results}

The simulated example concerns a road section of length $L=8[K m]$, with input/output flows $\left(\varphi_{\text {in }}, \varphi_{\text {out }}\right)$. The characteristic curve is represented by the constant values; $\left(w=16[\mathrm{Km} / \mathrm{h}], \rho_{m}=200[\mathrm{veh} / \mathrm{Km}]\right)$, while the section capacity and its associated critical density, $\varphi_{m}(v), \rho^{*}(v)$, are function of the selected speed limit $v$. The relaxation constant is $c=0.008[\mathrm{Km} / \mathrm{veh}]$. The control parameters are: the Dwell time $T_{s}=2[\mathrm{~min}]$, the variable speed limit set $\mathbb{U}=\left\{v_{\max }=110, v_{\min }=70\right\}[\mathrm{Km} / \mathrm{h}]$, and its associated steep change $\Delta_{v}=10[\mathrm{Km} / \mathrm{h}]$.

The input flow is selected as $\varphi_{\text {in }}=1800+200 \cos (15 t)$, and $\varphi_{\text {out }}=1800$. In this example there are phases where the input flow is larger than than output flow causing a congestion increase. There are also other phases when the congestion will tends to decrease. The objective here is to regulate the congestion front, as best as possible, to the value of $l_{r}=1[\mathrm{Km}]$. Simulation are shown in Fig. 4. The upper figure show the speed regulation values as produced by the control law. The lower curve shows the time-evolution of the 
congestion front when this controller is used, and compares the case when non speed limit regulation are used, and the regulation speed limit are fixed to $v=110[\mathrm{Km} / \mathrm{h}]$. It can be observed that the case with variable speed limits preserve the congestion close to the desired reference value.

\section{CONCLUSIONS}

In this paper we treated the problem of front congestions control. For this, we have introduced a new traffic lumped model with only two cells (one free, and another congested) the cells have variable length, and a variation law for the front congestion completes the 3-dimensional model. In opposition to fixed-length cell models that are commonly represented by a set of linear state-dependent switching systems, our model results in a lower dimensional nonlinear system which solutions are continuous.

Based on this model, we have designed a "best-effort" control strategy using variable speed limits. The notion of best effort control is here linked to the physical variable speed limit constraints which limits its size and as well as its rate variation. This results in a relative simple control in closed-form that can be implemented by using only information about the front congestion location.

\section{ACKNOWLEDGEMENTS}

Author will like to acknowledge the HYCON2 (Highlycomplex and networked control systems) EU-project from the ICT-FP7, and Denis Jacquet from Karrus-ITS for the discussion about the problem on front congestion control.

\section{REFERENCES}

[1] L.D. Baskar, B. De Schutter, and H. Hellendoorn, "Dynamic speed limits and on-ramp metering for IVHS using model predictive control," Proceedings of the 11th International IEEE Conference on Intelligent Transportation Systems (ITSC 2008), Beijing, China, pp. 821-826, Oct. 2008.

[2] C. F. Daganzo: The Cell Transmission Model: A Dynamic Representation of Highway Traffic Consistent with the Hydrodynamic Theory, Transportation Research Board, 28(4), 269-287, 1994.

[3] S. Godunov: A difference scheme for numerical solution of discontinuous solution of hydrodynamic equations, Matematicheskii Sbornik, 47, 271-306, 1959.

[4] G. Gomes, R. Horowitz, A. A. Kurzhanskiy, J. Kwon, and P. Varaiya: Behavior of the Cell Transmission Model and Effectiveness of Ramp Metering, Transportation Research, C, 16(4),485-513, 2008.

[5] G. Gomes, R. Horowitz: Optimal freeway ramp meetering using the asymetric cell transmission model, Transportation Research, C, 14, 244-262, 2006.

[6] A. Hegyi, B. De Schutter, and J. Hellendoorn, ”Optimal coordination of variable speed limits to suppress shock waves," IEEE Transactions on Intelligent Transportation Systems, vol. 6, no. 1, pp. 102-112, Mar. 2005.

[7] Jacquet D., Canudas De Wit C., Koenig D.: Optimal Control of Systems of Conservation Laws and Application to Non-Equilibrium Traffic Control, Proceedings of the 13th IFAC Workshop on Control Applications of Optimisation, France (2006)

[8] Jacquet D., Jaglin J., Koenig D., Canudas De Wit C.: Non-Local Feedback Ramp Metering Controller Design, Proceedings of the 11th IFAC Symposium on Control in Transportation Systems, CTS, Netherlands (2006)

[9] Koenig D., Jacquet D., Canudas De Wit C.: Optimal ramp metering strategy with extended LWR model, analysis and computational methods, Preprints of the 17th IFAC World Congress - IFAC World Congress, Czech Republic (2005)
[10] R.J. LeVeque: Numerical methods for conservation laws, Birkhäuser, 1992.

[11] M. J. Lighthill and G. B. Whitham: On kinematic waves II: A theory of traffic flow on long crowded roads, Proceedings of the Royal Society of London. Series A, Mathematical and Physical Sciences, 229(1178), 317-345, 1955.

[12] W.-H. Lin and D. Ahanotu: Validating the Basic Cell Transmission Model on a Single Freeway Link, PATH Technical Note 95-3, Institute of Transportation Studies, University of California at Berkeley, 1994

[13] P.-W. Lin and K.-P. Kang, and G.-L. Chang: Exploring the effectivness of variable speed limit control on highways work-zone operation. International Journal of Intelligent Transportation Systems, Vol.8, p155-168, 2004.

[14] X.-Y. Lu, P. Varaiya, R. Horowitz, D. Su, S. Shladover: “ A new approach for combined freeway variable speed limits and coordinated ramp metering", 13th International IEEE Conference on INtelligent Transportation Systems. madeira Island, Portugal, Sept. 12-22, (2010).

[15] M. Papageorgiou: Freeway ramp metering: an overview, IEEE Transactions on Intelligent Transportation Systems, Vol.3, No.4 271-281, 2002.

[16] I. Papamichail, I. Kampitaki, M. Papageorgiou, and A. Massmer: "Integrated ramp metering and variable speed limit control of motorway traffic flow. Preprints of the 17th IFAC World Congress, Seoul, Korea (2008).

[17] P. I. Richards: Shock Waves on the Highway, Operations Research, 4(1), 42-51, 1956. 